\title{
Effects of Cerebellar Transcranial Direct Current Stimulation on Upper Limb Motor Function After Stroke: Study Protocol for the Pilot of a Randomized Controlled Trial
}

\author{
Akiko Yuasa \\ Fujita Health University https://orcid.org/0000-0003-1808-2369 \\ Shintaro Uehara \\ Fujita Health University \\ Kazuki Ushizawa \\ Fujita Health University \\ Takamichi Toyama \\ Fujita Health University \\ Jose Gomez-Tames \\ Nagoya Institute of Technology \\ Akimasa Hirata \\ Nagoya Institute of Technology \\ Yohei Otaka ( $\sim$ otaka119@mac.com ) \\ Fujita Health University School of Medicine https://orcid.org/0000-0002-6797-2782
}

\section{Study Protocol}

Keywords: Transcranial direct current stimulation (tDCS), Stroke, Constraint-induced movement therapy (CIMT), Cerebellum

Posted Date: November 10th, 2021

DOI: https://doi.org/10.21203/rs.3.rs-1030199/v1

License: @ (1) This work is licensed under a Creative Commons Attribution 4.0 International License. Read Full License 


\section{Abstract}

Background: Transcranial direct current stimulation (tDCS) is a technique that can noninvasively modulate neural states in a targeted brain region. As cerebellar activity levels are associated with upper limb motor improvement after stroke, the cerebellum is a plausible target of tDCS. However, the effect of tDCS remains unclear. Here, we designed a pilot study to assess: 1) the feasibility of a study that aims to examine the effects of cerebellar tDCS combined with an intensive rehabilitation approach based on the concept of constraint-induced movement therapy (CIMT), and 2) the preliminary outcome of the combined approach on upper limb motor function in patients with stroke in the chronic stage.

Methods: This pilot study has a double-blind randomized controlled design. Twenty-four chronic stroke patients with mild to moderate levels of upper limb motor impairment will be randomly assigned to an active or sham tDCS group. The participants will receive 20 min of active or sham tDCS to the contralesional cerebellum at the commencement of 4 hours of daily intensive training, repeatedly for 5 days per week for 2 weeks. The primary outcome is upper limb motor function which will be evaluated using the Action Research Arm Test. Secondary outcomes comprise scores of the Fugl-Meyer Assessment for the upper extremity and the Motor Activity Log. Additionally, neurophysiological and neuroanatomical assessments of the cerebellum will be performed using transcranial magnetic stimulation and magnetic resonance imaging. These assessments will be conducted before, at the middle, and after the 2-week intervention, and finally 1 month after the intervention. Any adverse events that occur during the study will be recorded.

Discussion: Cerebellar tDCS combined with intensive upper limb training may increase the gains of motor improvement when compared to the sham condition. The present study should provide valuable evidence regarding the feasibility of the design and the efficacy of cerebellar tDCS for upper limb motor function in patients with stroke before a future large trial is conducted.

Trial registration: This study has been registered at the Japan Registry of Clinical Trials (jRCTs042200078; https://jrct.niph.go.jp/enlatest-detail/jRCTs042200078). Registered 17 December 2020

\section{Background}

Functional recovery of the upper limb is one of the primary goals in those who suffer stroke. In the last two decades, transcranial direct current stimulation (tDCS) has been widely investigated as a noninvasive brain stimulation technique that can enhance rehabilitation outcomes along with the modulation of brain activity [1]. Several studies using functional magnetic resonance imaging (MRI) [2,3] or transcranial magnetic stimulation (TMS) [4-7] have shown that tDCS has the potential to enhance neural plasticity. Many studies have attempted to facilitate motor recovery after stroke by applying tDCS to the primary motor cortex (M1) $[8,9]$. However, there is no consensus among systematic reviews regarding the effectiveness of tDCS for motor function after stroke. For instance, a recent review suggested that tDCS has significant effects on upper limb motor function in patients with stroke in the chronic stage [8]. However, a network meta-analysis, which synthesized the effects of multiple interventions (i.e., anodal, cathodal, and bilateral tDCS), showed that only cathodal tDCS had moderate effects on levels of activities of daily living, while no significant effects on the upper limb motor function were noted [9].

Recently, the neuromodulation of the cerebellum has gained increased attention. The cerebellum has multiple connections with motor and non-motor cerebral cortices and plays an important role as a hub of the brain network $[10,11]$. Among a wide range of cerebellar functions, its contribution to the motor domain, especially the learning processes, would be essential to patients after stroke [12,13]. Several imaging studies have suggested that increased cerebellar activity is associated with motor function improvement after stroke [14-18]. On the other hand, cerebellar inactivity such as crossed cerebellar diaschisis, which is caused by decreased blood flow and metabolism in the cerebellar hemisphere due to decreased neural inputs after stroke, is associated with functional impairments $[19,20]$. Furthermore, it has been reported that functional connectivity between M1 and the cerebellum $[11,21]$ and the structural integrity of the cortico-cerebellar tract $[17,18]$ seem to be related to motor recovery after stroke. These findings demonstrate the importance of the cerebellum and its functional connectivity to motor cortices, indicating that upregulating cerebellar neural activity would be beneficial for motor recovery after stroke [22,23]. Supporting this hypothesis, animal studies reported that, compared to sham stimulation, applying deep brain stimulation to the cerebellum in stroke-model rats resulted in superior motor improvement in association with neuroplastic changes in the area around a lesion [24-26]. Considering these findings, the cerebellum is a promising target for neuromodulation to facilitate improvements in motor function after stroke. 
In stroke rehabilitation, intensive task-oriented training is crucial for facilitating motor recovery. Constraint-induced movement therapy (CIMT) is a well-known approach for improving upper limb motor function after stroke. The key element in CIMT is that it encourages the use of the affected arm through intensive functionally oriented tasks [27,28]. Although it is generally accepted that neural and functional recovery reaches a plateau around 6 months after stroke onset [29], reviews have shown the effectiveness of CIMT in poststroke survivors in the chronic stage [30,31]. In addition, several stroke guidelines highly recommend CIMT as a beneficial approach for improving upper limb motor function and activity especially in the chronic stage [32,33]. Furthermore, studies reported that CIMT combined with tDCS [34,35] or combined with tDCS and the peripheral electrical stimulation [36] of motor-related cerebral regions induced greater effects on upper limb motor function compared to CIMT alone.

In view of these, we hypothesized that a combination of cerebellar tDCS and intensive movement therapy would further facilitate the improvement of motor function in stroke patients in the chronic stage. To clarify this hypothesis, we designed the present study to assess: 1 ) the feasibility of a study that will examine the effects of cerebellar tDCS combined with intensive upper limb training, and 2) the preliminary effects of this combined approach on upper limb motor function. The present study details a pilot trial that will be conducted before a large-scale randomized controlled trial.

\section{Methods/design Study design}

This study is a double-blind randomized controlled pilot trial and was designed according to the Consolidated Standards of Reporting Trials (CONSORT) extension to pilot trials [37]. The study flowchart is shown in Fig. 1. Twenty-four stroke patients will be enrolled and randomly allocated to an active or sham tDCS group. This study will be conducted in Fujita Health University Hospital, Aichi, Japan. All participants will provide written informed consent before participation according to the Declaration of Helsinki of 1964, as revised in 2013. This study has been approved by the Certified Review Board at Fujita Health University (approval No. CR21-029) and registered at the Japan Registry of Clinical Trials (jRCTs042200078).

\section{Participants}

The inclusion criteria are as follows: (1) age 40-79 years, (2) first-ever unilateral supratentorial ischemic or hemorrhagic stroke, (3) time exceeding 6 months after stroke onset, (4) mild to moderate upper limb hemiparesis [able to perform at least one component of the Action Research Arm Test (ARAT) in the grasp, grip, or pinch subscales; score of 10-51 on ARAT], and (5) independent walking ability [score of $\geq 6$ on the Functional Independence Measure (FIM) for walk]. The exclusion criteria are as follows: (1) unstable physical condition, (2) inability to communicate due to severe language impairment, (3) neurological disorders other than stroke, (4) facial sensory deficits, (5) inability to perform training because of psychological disorders or cognitive dysfunction, (6) cognitive impairments [Mini-Mental State Examination (MMSE) < 24] [38,39], (7) inability to perform training because of musculoskeletal disorders, (8) botulinum toxin injections into the arm within the last 6 months, and (9) any contraindications to tDCS, TMS, and MRI such as a history of epilepsy, metallic implants, cardiac pacemaker, drug or alcohol abuse, or pregnancy.

\section{Recruitment}

Potential participants will be identified from the outpatient clinic of the University Hospital and the local community. The advertisement for the study is open to the public via our department website to allow patients in the local community to access relevant information. Individuals who are interested in this study will be required to make an appointment for screening. The eligibility of potential participants will first be carefully assessed by a physician. They will then be screened for motor and cognitive functions using the ARAT and MMSE by a trained occupational therapist. Written informed consent will be obtained from those who fulfill the criteria and are willing to take part in this study.

\section{Randomization}

Participants will be stratified according to age and ARAT score, and randomly allocated to either the active tDCS or sham tDCS group using a computer-generated, permuted block randomization method with permuted block sizes of 2 and 4 . The random allocation will 
be conducted using the Research Electronic Data Capture (REDCap) tools [40] by independent researchers who will not be involved in any intervention and assessments.

\section{Interventions}

A time schedule of this study is shown in Fig. 2A. The participants will be admitted to Fujita Health University Hospital. The participants will undergo intensive upper limb training for 4 hours per day ( 2 hours per half-day) and receive cerebellar tDCS (active or sham) for $20 \mathrm{~min}$ at the beginning of the intensive training (Fig. 2B), considering that the effects of tDCS on the neural excitability of a targeted brain region last for 30-120 min [2,5-7]. This will be repeated for 5 consecutive days per week for 2 weeks (i.e., 10 days of intervention). During the intervention period, botulinum toxin injections and any changes of medications that could affect spasticity will not be allowed.

\section{tDCS}

The stimulation will be delivered using DC STIMULATOR PLUS (NeuroConn, GmbH, Ilmenau, Germany). The anode $(5 \times 5 \mathrm{~cm})$ will be placed on the cerebellar hemisphere on the contralesional side. Specifically, lobule VI, one of the subregions contributing to motor control of the upper limb [41], will be a focal target of the stimulation. Conventionally, the anode is placed at the fixed position $3 \mathrm{~cm}$ lateral to the inion [42]; however, in the present study, the optimal location for targeting the lobule VI will be determined for each individual using computational simulation (see Computational modeling subsection). The cathode $(5 \times 5 \mathrm{~cm})$ will be placed on the cheek on the contralesional side.

The detailed time course of active and sham tDCS is shown in Fig. 2C. The 'study mode' of the NeuroConn stimulator will be used to successfully implement double-blinding using 5-digit codes that activate the active or sham stimulation. Only the independent researchers that conduct the random allocation can access the code list. The experimenter will remain blinded to the information regarding which code belongs to the active or sham stimulation. In the active tDCS group, $1 \mathrm{~mA}$ constant current will be delivered for $20 \mathrm{~min}$, whereas in the sham tDCS group, $1 \mathrm{~mA}$ constant current will be delivered for $40 \mathrm{~s}$ to induce similar scalp sensations. In both groups, the current will be ramped up to $1 \mathrm{~mA}$ and ramped down over $15 \mathrm{~s}$ at the start and end of the stimulation [43]. During the sham tDCS condition, only a weak current of $110 \mu \mathrm{A}$ will be delivered every $550 \mathrm{~ms}$ after the ramped-down periods to test the electrode impedance. Only the impedance level and stimulation duration will be displayed in both conditions, which facilitates the blinding of the experimenter. The sham procedure is commonly used in tDCS research and at least 30-s active stimulation with slow ramp up and down are recommended for effective blinding during sham sessions [44]. The participants will not be given any information about stimulation parameters.

\section{Intensive upper limb training}

Intensive upper limb training based on the concept of CIMT will be provided by trained occupational therapists who will be blinded to the intervention allocation. CIMT is a therapeutic package that consists of repetitive and task-oriented training, behavioral methods for transferring the use of the affected arm in life situations, and constraining the use of the less affected arm to achieve participants' specific goals [45]. Since previous studies have reported that physical restraint has no significant effects on the outcome of CIMT $[46,47]$, no restraints will be applied to the unaffected arm of the participants in this study. Another difference compared to the original CIMT is with respect to the training hours per day, with 4 hours in this study compared to 6 hours in the original [27]. To accomplish sufficient training, participants will be encouraged to use their affected arm in activities of daily living. Additionally, the participants will engage in self-training programmed by the occupational therapists for 2 hours per day (Fig. 2B). If necessary, functional electrical stimulation will be provided to enhance muscle contractions and perform functional tasks, but rehabilitative robots will not be allowed.

\section{Computational modeling}

Fig. 3 shows the method that will be used to optimize the location of the tDCS montage at the individual level. First, the head model will be treated as a passive volume conductor constructed from 3D T1- and T2-weighted MRI of each participant before the experiment. The computational method has been reported in detail in a previous report $[48,49]$. It involves using the scalar potential 
finite difference method with fast computational techniques to accelerate the computational assessment before the intervention $[50,51]$. The computational model estimates the individualized electric field intensity and distribution on the cerebellar region applying a bipolar montage throughout the electrodes $(5 \times 5 \mathrm{~cm})$ with an injection current of $1 \mathrm{~mA}$, as used in the experiment. The cathode will be fixed on the buccinator muscle on the cheek on the contralesional side to avoid high electric currents flowing on the cerebrum. The anode will be placed on a grid $(13 \times 11$, size of grid $=10 \mathrm{~mm})$ centered on the inion to identify the position that generates the highest electric field strength (maximum and average values), with lobule VI as the target area. The optimal electrode location will be determined based on a reference landmark (10-20 system) and the Montreal Neurological Institute coordinates for confirmation.

\section{Outcomes}

The schedule of data collection is summarized in SPIRIT figure (Fig. 4). Clinical assessments of the upper limb and neurophysiological and neuroanatomical assessments will be conducted prior to the intervention (baseline, $\mathrm{t}_{0}$ ), at the middle of the intervention [middle, $t_{1}$ (ARAT only)], immediately after the intervention (post, $t_{2}$ ), and 1 month after the intervention (follow-up, $t_{3}$ ).

\section{Clinical assessments}

The primary outcome in this study is the ARAT score. ARAT is a clinical measurement that consists of 19 tests with 4 subscales: grasp, grip, pinch, and gross movement, which assess upper limb motor function [52,53]. The quality of movement is scored on an ordinal 4-point scale, ranging from 0 to 3 in each test, with a maximum score of 57 [52]. ARAT is classified as an outcome measurement of activity capacity [54,55] based on the International Classification of Functioning framework [56], and it shows excellent validity and reliability in chronic stroke patients [57].

Secondary outcomes include the Fugl-Meyer Assessment for the upper extremity (FMA) and Motor Activity Log-14 (MAL-14). FMA is classified as an outcome measurement of body functions [55] based on the International Classification of Functioning framework [56]. FMA for the upper extremity consists of 33 items including reflex testing, movement observation, grasp testing, and coordination assessment. The score ranges from 0 to 66 . FMA shows excellent validity and reliability in chronic stroke patients [58,59]. MAL-14 is a structured interview to assess how much (amount of use: AOU) and how well (quality of movement: QOM) a patient uses the paretic hand and arm during activities of daily living [60,61]. For the AOU assessment, the score ranges from 0 (never uses the arm) to 5 (uses the arm as often as before the stroke), while for the QOM assessment, the score ranges from 0 (never uses the arm) to 5 (uses the arm as well as before the stroke). MAL-14 is classified as an outcome measurement of activity performance $[54,55]$ based on the International Classification of Functioning framework [56], and it shows excellent validity and reliability in chronic stroke patients [62].

ARAT and FMA will be video-recorded [63], and video-based assessments will be conducted by two trained occupational therapists who will be blinded to the allocation and will not be involved in the interventions. In addition, the following participant baseline characteristics will be collected: age, affected side of the brain, time since onset, and FIM and MMSE scores.

\section{Neurophysiological assessments}

As an index of cerebellar excitability, we will assess the magnitude of cerebellar inhibition (CBI) to the contralateral M1 using a pairedpulse TMS paradigm (Magstim 200², Magstim Company, Whitland, UK) (Fig. 5). CBI will be measured by delivering a conditioning stimulus (CS) over the cerebellum for 5 ms prior to a test stimulus (TS) over M1, resulting in the reduction of motor evoked potential (MEP) elicited by TS over M1[64]. CBI is thought to be driven by inhibitory outputs from the cerebellar cortex to the deep cerebellar nuclei that have excitatory outputs to the contralateral M1 [65]. Therefore, changes in CBI magnitude can be interpreted as cerebellar excitability changes $[66,67]$. We will assess the cerebellar excitability changes with the assumption that cerebellar plasticity may underlie the intervention effects.

The CS will be delivered to the contralesional side of the cerebellum using a double-cone coil which will be centered at the same location determined by the computation modeling, while the TS will be delivered to the ipsilesional side of M1 using the figure-of-eight coil over the optimal stimulation site ("hot spot") of the first dorsal interosseous (FDI) muscle (Fig. 5). To determine the intensity of the cerebellar CS, we will first test the brainstem active motor threshold for the pyramidal tract by delivering stimuli over the inion using a single pulse with the double-cone coil. The brainstem active motor threshold is defined as the nearest $5 \%$ maximum stimulator output 
(MSO) that elicits MEPs exceeding $50 \mu \mathrm{V}$ from the FDI muscle in the affected hand in at least 5 of 10 successive stimuli [68]. The cerebellar CS intensity will be set at $5 \%$ below the brainstem active motor threshold $[64,69,70]$. If the threshold is not observed below $80 \%$ of MSO, $70 \%$ of MSO will be used for the cerebellar CS. For the intensity of TS over M1, we will first assess the resting motor threshold (rMT) of the FDI muscle which is determined as the lowest intensity that evokes an MEP amplitude greater than $50 \mu \mathrm{V}$ in at least 5 of 10 successive stimuli [68]. Thereafter, the TS intensity will be set at $125 \%$ of rMT. For the CBI assessment, in a set of 30 TS, 15 TS will be combined with the preceding CS (conditioned TS), while the other 15 TS will be delivered without CS (unconditioned TS). The inter-stimulus interval for TS will be 4-6s, and the order of conditioned and unconditioned TS will be randomized. The position of the TS over M1 will be tracked using a neuronavigation system (Brainsight, Rogue Research, Montreal, Canada). CBI will be calculated as the ratio of the mean amplitude of conditioned MEP over the unconditioned MEP.

We will also assess M1 excitability by delivering single-pulse TMS over the ipsilesional M1 as a separate measurement set to confirm that potential $\mathrm{CBI}$ changes will not be accompanied by M1 excitability changes. For this purpose, 15 stimuli with an intensity of $125 \%$ of rMT will be applied with the inter-stimulus interval of $4-6 \mathrm{~s}$. The mean amplitude will be used as a proxy for M1 excitability.

Surface electromyography will be recorded using a biosignal recording system (Nuropack X1 MEB-2312; Nihon Kohden Corporation, Tokyo, Japan) at the frequency of $5 \mathrm{kHz}$, with a bandpass filter of $10 \mathrm{~Hz}$ to $10 \mathrm{kHz}$. Recorded analog data will be digitized with a micro 1401 AD converter (Cambridge Electronic Design, Cambridge, UK) and stored on a computer (Signal Software, Cambridge Electronic Design, Cambridge, UK) for offline analysis.

\section{MRI}

To identify structural characteristics of the individual brain, MR images will be acquired prior to the intervention (baseline, $\mathrm{t}_{0}$ ) and after the intervention (post, $\mathrm{t}_{2}$ ). The brain MRI will be conducted using a 3-T scanner (Vantage Centurian 3T; Canon Medical Systems, Tochigi, Japan). For brain tissue segmentation, 3D T1- and T2-weighted images with high resolution (voxel size: $0.8 \times 0.8 \times 0.8 \mathrm{~mm}^{3}$ ) will be acquired [71]. In addition, to identify functional and structural connectivity between widespread brain areas, resting-state functional MRI and diffusion tensor imaging will be obtained [72]. The 3D T1- and T2-weighted images will be used for the computational modeling (see Computational Modeling subsection).

\section{Safety evaluation}

To evaluate the safety of the intervention and overall protocol, we will record adverse events such as burns to the skin, prolonged abnormal cutaneous sensation, dizziness, fatigue, pain related to overuse of the affected arm, etc. To monitor the existence of the temporary side effects, especially of the tDCS, we will provide participants with a questionnaire [73] after every 20 min of active or sham tDCS intervention. The questionnaire consists of 14 items regarding symptoms which will enable us to determine if the participants are experiencing severe symptoms. The score ranges from 1 to 10 , from absent to severe symptoms.

\section{Feasibility criteria}

We created the feasibility criteria based on the suggested reasons for conducting a pilot study [74,75]. We adapted the criteria to our study settings as follows: (1) rates of eligible participants during the screening, (2) retention and follow-up rates throughout the study, (3) adherence rate to the study protocol (amount of total training hours of each participant), and (4) validity of the data collection schedule.

\section{Sample size}

A previous study proposed the rules of thumb for estimating the sample size of a pilot study based on the anticipated effect size of the future main trial [76]. According to the proposal, if the future main trial is designed around medium to large effect with $80 \%$ power and a two-sided $5 \%$ significance, a sample size for the pilot trial is set at 10 in each group [76]. Allowing for a $20 \%$ of drop-out rate, we will recruit 12 participants in each group. 


\section{Blinding}

Participants, assessors, occupational therapists providing intensive upper limb training, experimenter applying the tDCS, and a researcher analyzing the data will be all blinded to group allocation. Independent researchers who will conduct the random allocation will not be blinded. A researcher who is in charge of patients' safety and risk management can access the group allocation via the REDCap if necessary.

\section{Analytical methods}

The active and sham tDCS groups will be compared with regard to demographic variables to assess group differences using the Student $t$-test or Mann-Whitney Utest for continuous variables and the $\chi^{2}$ test for categorical variables. Group differences of the primary (ARAT score) and secondary (FMA, MAL-14, and neurophysiological measures) outcomes will be analyzed using multivariable regression analyses and compared at each assessment time point. Furthermore, adverse events and their frequency and the questionnaire score will be reported to evaluate the safety of the procedure.

\section{Discussion}

Neuromodulatory intervention for the cerebellum has been suggested to improve motor function of stroke survivors in the chronic stage. Our pilot trial aims to assess the feasibility of the proposed study (e.g., outcome measures, adverse effects, and follow-up rates, etc.) and the efficacy (i.e., effect size) of the application of tDCS to the cerebellum on upper limb motor function in patients after stroke. A future large-scale randomized controlled trial will be performed based on the results of the present study.

The present study has some potential limitations. First, the study aims to enroll patients with mild to moderate hemiparesis since intensive upper limb training requires voluntary movements of the affected hand to some extent. Therefore, the findings need to be carefully translated into clinical practice especially when considering the intervention effects in severe cases. Second, in conventional cerebellar tDCS, the anode is placed at a fixed position $3 \mathrm{~cm}$ lateral to the inion [42], whereas in the present study, the optimal electrode location will be determined for each individual using computational simulation. However, it remains unclear whether this personalized tDCS maneuver will improve outcomes regarding upper limb motor function compared to conventional tDCS. This question should be addressed in another future study.

To our knowledge, this is the first series of studies to evaluate the effects of cerebellar tDCS combined with intensive training on upper limb motor function in chronic stroke patients. We believe that cerebellar tDCS will improve the effect of intensive upper limb training. The comprehensive results from the present study and the forthcoming large-scale trial should provide significant evidence regarding cerebellar tDCS to determine whether it can be a beneficial intervention for stroke patients.

\section{Abbreviations}

AOU, amount of use; ARAT, Action Research Arm Test; CBI, cerebellar brain inhibition; CIMT, constraint-induced movement therapy; CS, conditioning stimulus; FDI, first dorsal interosseous; FIM, functional independence measure; FMA, Fugl-Meyer assessment for the upper extremity; M1, primary motor cortex; MAL-14, Motor Activity Log-14; MEP, motor-evoked potential; MMSE, Mini-Mental State Examination; MR/MRI, magnetic resonance / magnetic resonance imaging; MSO, maximum stimulator output; QOM, quality of movement; tDCS, transcranial direct current stimulation; TMS, transcranial magnetic stimulation; TS, test stimulus

\section{Declarations}

\section{Ethics approval and consent to participate}

Approval was obtained from the Certified Review Board at Fujita Health University (approval No. CR21-029). The study was registered at the Japan Registry of Clinical Trials (jRCTs042200078). Participants will be required to provide informed consent before inclusion into the study.

\section{Consent for publication}


Not applicable.

\section{Availability of data and materials}

The datasets used and/or analyzed during the current study are available from the corresponding author on reasonable request.

\section{Competing interests}

The authors declare that they have no competing interests.

\section{Funding}

Not applicable.

\section{Authors' contributions}

YO, SU, and AH conceived the study. AY, SU, and YO established the detailed protocol. TT and KU contributed to the refinement of the study protocol. AY, SU, GJ, and TT wrote the manuscript. YO supervised the project. All authors read and approved the final manuscript.

\section{Acknowledgements}

We would like to thank Mr. Takuya Suzuki, Mr. Kazuki Ito, Mr. Yota Obayashi, and Mr. Shotaro Furuzawa for their assistance in planning the study protocol. We would like to also thank Mr. Nobuhiro Kumazawa, Mr. Takuma li, and Mr. Hikaru Kondo for their support in designing the randomization method.

\section{References}

1. Lefaucheur JP, Antal A, Ayache SS, Benninger DH, Brunelin J, Cogiamanian F, et al. Evidence-based guidelines on the therapeutic use of transcranial direct current stimulation (tDCS). Clinical Neurophysiology. 2017;128(1):56-92. https://doi.org/10.1016/j.clinph.2016.10.087

2. Jamil A, Batsikadze G, Kuo HI, Meesen RLJ, Dechent P, Paulus W, et al. Current intensity- and polarity-specific online and aftereffects of transcranial direct current stimulation: An fMRI study. Hum Brain Mapp. 2020 Apr 15;41(6):1644-66. https://doi.org/10.1002/hbm.24901

3. McCambridge AB, Stinear JW, Byblow WD. Revisiting interhemispheric imbalance in chronic stroke: A tDCS study. Clin Neurophysiol. 2018 Jan 1;129(1):42-50. https://doi.org/10.1016/j.clinph.2017.10.016

4. Nitsche MA, Paulus W. Excitability changes induced in the human motor cortex by weak transcranial direct current stimulation. $J$ Physiol. 2000;527(3):633-9. https://doi.org/10.1111/j.1469-7793.2000.t01-1-00633.x

5. Nitsche MA, Paulus W. Sustained excitability elevations induced by transcranial DC motor cortex stimulation in humans. Neurology. 2001 Nov 27;57(10):1899 - 1901. https://doi.org/10.1212/WNL.57.10.1899

6. Jamil A, Batsikadze G, Kuo HI, Labruna L, Hasan A, Paulus W, et al. Systematic evaluation of the impact of stimulation intensity on neuroplastic after-effects induced by transcranial direct current stimulation. J Physiol. 2017 Feb 15;595(4):1273-88. https://doi.org/10.1113/JP272738

7. Agboada D, Mosayebi-Samani M, Kuo MF, Nitsche MA. Induction of long-term potentiation-like plasticity in the primary motor cortex with repeated anodal transcranial direct current stimulation - Better effects with intensified protocols? Brain Stimul. 2020 Jul 1;13(4):987-97. https://doi.org/10.1016/j.brs.2020.04.009

8. Bai X, Guo Z, He L, Ren L, McClure MA, Mu Q. Different Therapeutic Effects of Transcranial Direct Current Stimulation on Upper and Lower Limb Recovery of Stroke Patients with Motor Dysfunction: A Meta-Analysis. Vol. 2019, Neural Plasticity. Hindawi Limited; 2019. https://doi.org/10.1155/2019/1372138

Page 8/16 
9. Elsner B, Kwakkel G, Kugler J, Mehrholz J. Transcranial direct current stimulation (tDCS) for improving capacity in activities and arm function after stroke: A network meta-analysis of randomised controlled trials. Journal of NeuroEngineering and Rehabilitation. BioMed Central Ltd.; 2017;14(1). https://doi.org/10.1186/s12984-017-0301-7

10. Stoodley CJ, Schmahmann JD. Functional topography in the human cerebellum: A meta-analysis of neuroimaging studies. Neuroimage. 2009 Jan 15;44(2):489-501. https://doi.org/10.1016/j.neuroimage.2008.08.039

11. Wang L, Yu C, Chen H, Qin W, He Y, Fan F, et al. Dynamic functional reorganization of the motor execution network after stroke. Brain. 2010 Apr;133(4):1224-38. https://doi.org/10.1093/brain/awq043

12. Askim T, Indredavik B, Vangberg T, Håberg A. Motor network changes associated with successful motor skill relearning after acute ischemic stroke: A longitudinal functional magnetic resonance imaging study. Neurorehabil Neural Repair. 2009 Mar;23(3):295304. https://doi.org/10.1177/1545968308322840

13. Manto M, Bower JM, Conforto AB, Delgado-García JM, Da Guarda SNF, Gerwig M, et al. Consensus paper: Roles of the cerebellum in motor control-the diversity of ideas on cerebellar involvement in movement. Cerebellum. 2012;11(2):457-87. https://doi.org/10.1007/s12311-011-0331-9

14. Ward NS, Brown MM, Thompson AJ, Frackowiak RSJ. Neural correlates of motor recovery after stroke: A longitudinal fMRI study. Brain. 2003;126(11):2476-96. https://doi.org/10.1093/brain/awg245

15. Small SL, Hlustik P, Noll DC, Genovese C, Solodkin A. Cerebellar hemispheric activation ipsilateral to the paretic hand correlates with functional recovery after stroke. Brain. 2002;125(7):1544-57. https://doi.org/10.1093/brain/awf148

16. Rehme AK, Eickhoff SB, Rottschy C, Fink GR, Grefkes C. Activation likelihood estimation meta-analysis of motor-related neural activity after stroke. Neuroimage. 2012 Feb 1;59(3):2771-82. https://doi.org/10.1016/j.neuroimage.2011.10.023

17. Schulz R, Frey BM, Koch P, Zimerman M, Bönstrup M, Feldheim J, et al. Cortico-cerebellar structural connectivity is related to residual motor output in chronic stroke. Cereb Cortex. 2017 Jan 1;27(1):635-45. https://doi.org/10.1093/cercor/bhv251

18. Guder S, Frey BM, Backhaus W, Braass H, Timmermann JE, Gerloff C, et al. The Influence of Cortico-Cerebellar Structural Connectivity on Cortical Excitability in Chronic Stroke. Cereb Cortex. 2020;30(3):1330-44. https://doi.org/10.1093/cercor/bhz169

19. Szilágyi G, Vas Á, Kerényi L, Nagy Z, Csiba L, Gulyás B. Correlation between crossed cerebellar diaschisis and clinical neurological scales. Acta Neurol Scand. 2012 Jun 1;125(6):373-81. https://doi.org/10.1111/j.1600-0404.2011.01576.x

20. Takasawa M, Watanabe M, Yamamoto S, Hoshi T, Sasaki T, Hashikawa K, et al. Prognostic value of subacute crossed cerebellar diaschisis: Single-photon emission CT study in patients with middle cerebral artery territory infarct. Am J Neuroradiol. 2002;23(2):189-93.

21. Rosso C, Valabregue R, Attal Y, Vargas P, Gaudron M, Baronnet F, et al. Contribution of Corticospinal Tract and Functional Connectivity in Hand Motor Impairment after Stroke. PLoS One. 2013;8(9):1-11. https://doi.org/10.1371/journal.pone.0073164

22. Wessel MJ, Hummel FC. Non-invasive Cerebellar Stimulation: a Promising Approach for Stroke Recovery? Vol. 17, Cerebellum. Springer New York LLC; 2018;17(3):359-71. https://doi.org/10.1007/s12311-017-0906-1

23. Miterko LN, Baker KB, Beckinghausen J, Bradnam L V., Cheng MY, Cooperrider J, et al. Consensus Paper: Experimental Neurostimulation of the Cerebellum. Cerebellum. 2019 Dec 1;18(6):1064-97. https://doi.org/10.1007/s12311-019-01041-5

24. MacHado AG, Cooperrider J, Furmaga HT, Baker KB, Park HJ, Chen Z, et al. Chronic 30-Hz deep cerebellar stimulation coupled with training enhances post-ischemia motor recovery and peri-infarct synaptophysin expression in rodents. Neurosurgery. 2013 Aug;73(2):344-53. https://doi.org/10.1227/01.neu.0000430766.80102.ac

25. Cooperrider J, Furmaga H, Plow E, Park HJ, Chen Z, Kidd G, et al. Chronic deep cerebellar stimulation promotes long-term potentiation, microstructural plasticity, and reorganization of perilesional cortical representation in a rodent model. J Neurosci. 2014;34(27):9040-50. https://doi.org/10.1523/JNEUROSCI.0953-14.2014

26. Baker KB, Schuster D, Cooperrider J, Machado AG. Deep brain stimulation of the lateral cerebellar nucleus produces frequencyspecific alterations in motor evoked potentials in the rat in vivo. Exp Neurol. 2010;226(2):259-64. http://dx.doi.org/10.1016/j.expneurol.2010.08.019

27. Taub E, Miller NE, Novack TA, Cook EW, Fleming WC, Nepomuceno CS, et al. Technique to improve chronic motor deficit after stroke. Arch Phys Med Rehabil. 1993;74(4):347-54.

28. Wolf SL, Blanton S, Baer H, Breshears J, Butler AJ. Repetitive task practice: a critical review of constraint-induced movement therapy in stroke. Neurologist. 2002 Nov;8(6):325-38. https://pubmed.ncbi.nlm.nih.gov/12801434 
29. Langhorne P, Bernhardt J, Kwakkel G. Stroke rehabilitation. Lancet. 2011 May 14 ;377(9778):1693-702. http://www.ncbi.nlm.nih.gov/pubmed/21571152

30. Corbetta D, Sirtori V, Castellini G, Moja L, Gatti R. Constraint-induced movement therapy for upper extremities in people with stroke. Vol. 2015, Cochrane Database of Systematic Reviews. John Wiley and Sons Ltd; 2015. p. CD004433. https://doi.org/10.1002/14651858.CD004433.pub3

31. Lin IH, Tsai HT, Wang CY, Hsu CY, Liou TH, Lin YN. Effectiveness and Superiority of Rehabilitative Treatments in Enhancing Motor Recovery Within 6 Months Poststroke: A Systemic Review. Archives of Physical Medicine and Rehabilitation. W.B. Saunders; 2019;100(2):366-78. https://doi.org/10.1016/j.apmr.2018.09.123

32. Winstein CJ, Stein J, Arena R, Bates B, Cherney LR, Cramer SC, et al. Guidelines for Adult Stroke Rehabilitation and Recovery: A Guideline for Healthcare Professionals from the American Heart Association/American Stroke Association. Stroke. 2016;47(6):e98-169. https://doi.org/10.1161/STR.0000000000000098

33. Teasell R, Hussein N, Mirkowski M, Rrt DV, Saikaley Hbsc M, Longval Bsc M, et al. Stroke Rehabilitation Clinician Handbook 4. Hemiplegic Upper Extremity Rehabilitation 2020. http://www.ebrsr.com/sites/default/files/EBRSR\%20Handbook\%20Chapter\%204_Upper\%20Extremity\%20Post\%20Stroke_ML.pdf. Accessed 20 July 2021.

34. Bolognini N, Vallar G, Casati C, Latif LA, El-Nazer R, Williams J, et al. Neurophysiological and behavioral effects of tDCS combined with constraint-induced movement therapy in poststroke patients. Neurorehabil Neural Repair. 2011 Nov;25(9):819-29. https://doi.org/10.1177/1545968311411056

35. Cunningham DA, Varnerin N, Machado A, Bonnett C, Janini D, Roelle S, et al. Stimulation targeting higher motor areas in stroke rehabilitation: A proof-of-concept, randomized, double-blinded placebo-controlled study of effectiveness and underlying mechanisms. Restor Neurol Neurosci. 2015 Nov 16;33(6):911-26. https://doi.org/10.3233/RNN-150574

36. Takebayashi T, Takahashi K, Moriwaki M, Sakamoto T, Domen K. Improvement of upper extremity deficit after constraint-induced movement therapy combined with and without preconditioning stimulation using dual-hemisphere transcranial direct current stimulation and peripheral neuromuscular stimulation in chronic stroke. Front Neurol. 2017 Oct 30;8(OCT). https://doi.org/10.3389/fneur.2017.00568

37. Eldridge SM, Chan CL, Campbell MJ, Bond CM, Hopewell S, Thabane L, et al. CONSORT 2010 statement: extension to randomised pilot and feasibility trials. Pilot Feasibility Stud. 2016 Dec 21;2(1):64. https://doi.org/10.1186/s40814-016-0105-8

38. Tsoi KKF, Chan JYC, Hirai HW, Wong SYS, Kwok TCY. Cognitive tests to detect dementia a systematic review and meta-analysis. JAMA Intern Med. 2015 Sep 1;175(9):1450-8. https://doi.org/10.1001/jamainternmed.2015.2152

39. Folstein MF, Folstein SE, Mchugh PR. "MINI-MENTAL STATE” A PRACTICAL METHOD FOR GRADING THE COGNITIVE STATE OF PATIENTS FOR THE CLINICIAN*. Vol. 12, J. gsychiaf. Res. Pergamon Press; 1975;12:189-198.

40. Harris PA, Taylor R, Minor BL, Elliott V, Fernandez M, O'Neal L, et al. The REDCap consortium: Building an international community of software platform partners. J Biomed Inform. 2019;95(May):103208. https://doi.org/10.1016/j.jbi.2019.103208

41. Mottolese C, Richard N, Harquel S, Szathmari A, Sirigu A, Desmurget M. Mapping motor representations in the human cerebellum. Brain. 2013;136(1):330-42. https://doi.org/10.1093/brain/aws186

42. Rampersad SM, Janssen AM, Lucka F, Aydin U, Lanfer B, Lew S, et al. Simulating transcranial direct current stimulation with a detailed anisotropic human head model. IEEE Trans Neural Syst Rehabil Eng. 2014;22(3):441-52. https://doi.org/10.1109/TNSRE.2014.2308997

43. Grimaldi G, Taib NO Ben, Manto M, Bodranghien F. Marked reduction of cerebellar deficits in upper limbs following transcranial cerebello-cerebral DC stimulation: Tremor reduction and re-programming of the timing of antagonist commands. Front Syst Neurosci. 2014;8(JAN):1-12. https://doi.org/10.3389/fnsys.2014.00009

44. van Dun K, Bodranghien FCAA, Mariën P, Manto MU. TDCS of the cerebellum: Where do we stand in 2016? Technical issues and critical review of the literature. Frontiers in Human Neuroscience. Frontiers Media S. A; 2016;10. https://doi.org/10.3389/fnhum.2016.00199

45. Morris DM, Taub E, Mark VW. Constraint-induced movement therapy: Characterizing the intervention protocol. Eura Medicophys. 2006;42(3):257-68.

46. Brogårdh C, Vestling M, Sjölund BH. Shortened constraint-induced movement therapy in subacute stroke - No effect of using a restraint: A randomized controlled study with independent observers. J Rehabil Med. 2009;41(4):231-6.

Page 10/16 
https://doi.org/10.2340/16501977-0312

47. Brunner IC, Skouen JS, Strand LI. Is modified constraint-induced movement therapy more effective than bimanual training in improving arm motor function in the subacute phase post stroke? A randomized controlled trial. Clin Rehabil. 2012;26(12):107886. https://doi.org/10.1177/0269215512443138

48. Gomez-Tames J, Sugiyama Y, Laakso I, Tanaka S, Koyama S, Sadato N, et al. Effect of microscopic modeling of skin in electrical and thermal analysis of transcranial direct current stimulation. Phys Med Biol. 2016;61(24):8825-38. https://doi.org/10.1088/1361-6560/61/24/8825

49. Laakso I, Tanaka S, Koyama S, De Santis V, Hirata A. Inter-subject Variability in Electric Fields of Motor Cortical tDCS. Brain Stimul. 8(5):906-13. https://www.sciencedirect.com/science/article/pii/S1935861X15009419

50. Dawson TW, Stuchly MA. High-resolution organ dosimetry for human exposure to low-frequency magnetic fields. IEEE Trans Magn. 1998;34(3):708-18. https://doi.org/10.1109/20.668071

51. Laakso I, Hirata A. Fast multigrid-based computation of the induced electric field for transcranial magnetic stimulation. Phys Med Biol. 2012;57(23):7753-65.

52. Yozbatiran N, Der-Yeghiaian L, Cramer SC. A standardized approach to performing the action research arm test. Neurorehabil Neural Repair. 2008 Jan;22(1):78-90. https://doi.org/10.1177/1545968307305353

53. Lyle RC. A performance test for assessment of upper limb function in physical rehabilitation treatment and research. Int $\mathrm{J}$ Rehabil Res 1981;4(4). https://journals.Iww.com/intjrehabilres/Fulltext/1981/12000/A_performance_test_for_assessment_of_upper_limb.1.aspx

54. Lemmens RJM, Timmermans AAA, Janssen-Potten YJM, Smeets RJEM, Seelen HAM. Valid and reliable instruments for arm-hand assessment at ICF activity level in persons with hemiplegia: A systematic review. BMC Neurol 12(1):21. https://doi.org/10.1186/1471-2377-12-21.

55. Salter K, Campbell N, Richardson M, Mehta S, Jutai J, Zettler L, et al. Outcome Measures in Stroke Rehabilitation. EBRSR. 2013. http://www.ebrsr.com/evidence-review/20-outcome-measures-stroke-rehabilitation. Accessed 10 July 2021.

56. World Health Organization. ICF Browser (2017 - English version) https://apps.who.int/classifications/icfbrowser/(2017). Accessed 25 Aug 2021.

57. Platz T, Pinkowski C, Berlin K. Reliability and validity of arm function assessment with standardized guidelines for the Fugl-Meyer Test , Action Research Arm Test and Box and Block Test: a multicentre study. Clin Rehabil. 2005;19:404-11. https://doi.org/10.1191/0269215505cr832oa.

58. Page SJ, Hade E, Persch A. Psychometrics of the Wrist Stability and Hand Mobility Subscales of the Fugl-Meyer Assessment in Moderately Impaired Stroke. Phys Ther. 2015;95(1):103-108. https://doi.org/10.2522/ptj.20130235

59. See J, Dodakian L, Chou C, Chan V, McKenzie A, Reinkensmeyer DJ, et al. A standardized approach to the Fugl-Meyer assessment and its implications for clinical trials. Neurorehabil Neural Repair. 2013 Oct;27(8):732-41.

https://doi.org/10.1177/1545968313491000

60. Lee JH Van Der, Beckerman H, Knol DL, Vet HCW De, Bouter LM. Clinimetric Properties of the Motor Activity Log for the Assessment of Arm Use in Hemiparetic Patients. Stroke. 2004;35(6):1410-4. https://doi.org/10.1161/01.STR.0000126900.24964.7e

61. Taub E, McCulloch K, Uswatte G, Morris DM. Motor Activity Log (MAL) Manual. Ther Res Gr. 2011;1-18. https://www.uab.edu/citherapy/images/pdf_files/CIT_Training_MAL_manual.pdf. Accessed 20 Apr 2021.

62. Uswatte G, Taub E, Morris D, Vignolo M, McCulloch K. Reliability and validity of the upper-extremity motor activity log-14 for measuring real-world arm use. Stroke. 2005 Nov;36(11):2493-6. https://doi.org/10.1161/01.STR.0000185928.90848.2e

63. Amano S, Umeji A, Uchita A, Hashimoto Y, Takebayashi T, Kanata Y, et al. Reliability of remote evaluation for the Fugl-Meyer assessment and the action research arm test in hemiparetic patients after stroke. Top Stroke Rehabil. 2018;25(6):432-7. https://doi.org/10.1080/10749357.2018.1481569

64. Ugawa Y, Uesaka Y, Terao Y, Hanajima R, Kanazawa I. Magnetic stimulation over the cerebellum in humans. Ann Neurol. 1995;37(6):703-13. https://doi.org/10.1002/ana.410370603

65. Grimaldi G, Argyropoulos GP, Bastian A, Cortes M, Davis NJ, Edwards DJ, et al. Cerebellar Transcranial Direct Current Stimulation (ctDCS): A Novel Approach to Understanding Cerebellar Function in Health and Disease. Neuroscientist. SAGE Publications Inc.; 2016;22(1):83-97. https://doi.org/10.1177/1073858414559409

Page $11 / 16$ 
66. Spampinato DA, Celnik PA, Rothwell JC. Cerebellar-Motor Cortex Connectivity: One or Two Different Networks? J Neurosci. 2020 Apr 20;40(21):4230-4239. https://doi.org/10.1523/JNEUROSCI.2397-19.2020

67. Uehara S, Mawase F, Celnik P. Learning similar actions by reinforcement or sensory-prediction errors rely on distinct physiological mechanisms. Cereb Cortex. 2018;28(10):3478-90. https://doi.org/10.1093/cercor/bhx214

68. Rossini PM, Burke D, Chen R, Cohen LG, Daskalakis Z, lorio R Di, et al. Non-invasive electrical and magnetic stimulation of the brain , spinal cord, roots and peripheral nerves: Basic principles and procedures for routine clinical and research application. An updated report from an I.F.C.N. Committee. Clin Neurophysiol. 2015;126(6):1071-107.

http://dx.doi.org/10.1016/j.clinph.2015.02.001

69. Batsikadze G, Rezaee Z, Chang D-I, Gerwig M, Herlitze S, Dutta A, et al. Effects of cerebellar transcranial direct current stimulation on cerebellar-brain inhibition in humans: A systematic evaluation. Brain Stimul. 2019;12(5):1177-86.

https://doi.org/10.1016/j.brs.2019.04.010

70. Pinto AD, Chen R. Suppression of the motor cortex by magnetic stimulation of the cerebellum. Exp Brain Res. 2001;140(4):50510. https://doi.org/10.1007/s002210100862

71. Mori S, Zhang J. Principles of Diffusion Tensor Imaging and Its Applications to Basic Neuroscience Research. Neuron. 2006;51(5):527-39. https://doi.org/10.1016/j.neuron.2006.08.012

72. Carter AR, Patel BA KR, Astafiev S V, Snyder AZ, Rengachary J, Strube MJ, et al. Upstream Dysfunction of Somatomotor Functional Connectivity After Corticospinal Damage in Stroke. Neurorehabil Neural Repair. 2012;26(1):7-19. https://doi.org/10.1177/1545968311411054

73. Thair H, Holloway AL, Newport R, Smith AD. Transcranial direct current stimulation (tDCS): A Beginner's guide for design and implementation. Front Neurosci. 2017 Nov 22;11(NOV). https://doi.org/10.3389/fnins.2017.00641

74. Thabane L, Ma J, Chu R, Cheng J, Ismaila A, Rios LP, et al. A tutorial on pilot studies: the what, why and how. BMC Med Res Methodol. 2010;10(1):1. https://doi.org/10.1186/1471-2288-10-1

75. Orsmond GI, Cohn ES. The distinctive features of a feasibility study: Objectives and guiding questions. OTJR Occup Particip Heal. 2015;35(3):169-77. https://doi.org/10.1177/1539449215578649

76. Whitehead AL, Julious SA, Cooper CL, Campbell MJ. Estimating the sample size for a pilot randomised trial to minimise the overall trial sample size for the external pilot and main trial for a continuous outcome variable. Stat Methods Med Res. 2015;25(3):1057-73. https://doi.org/10.1177/0962280215588241

\section{Figures}




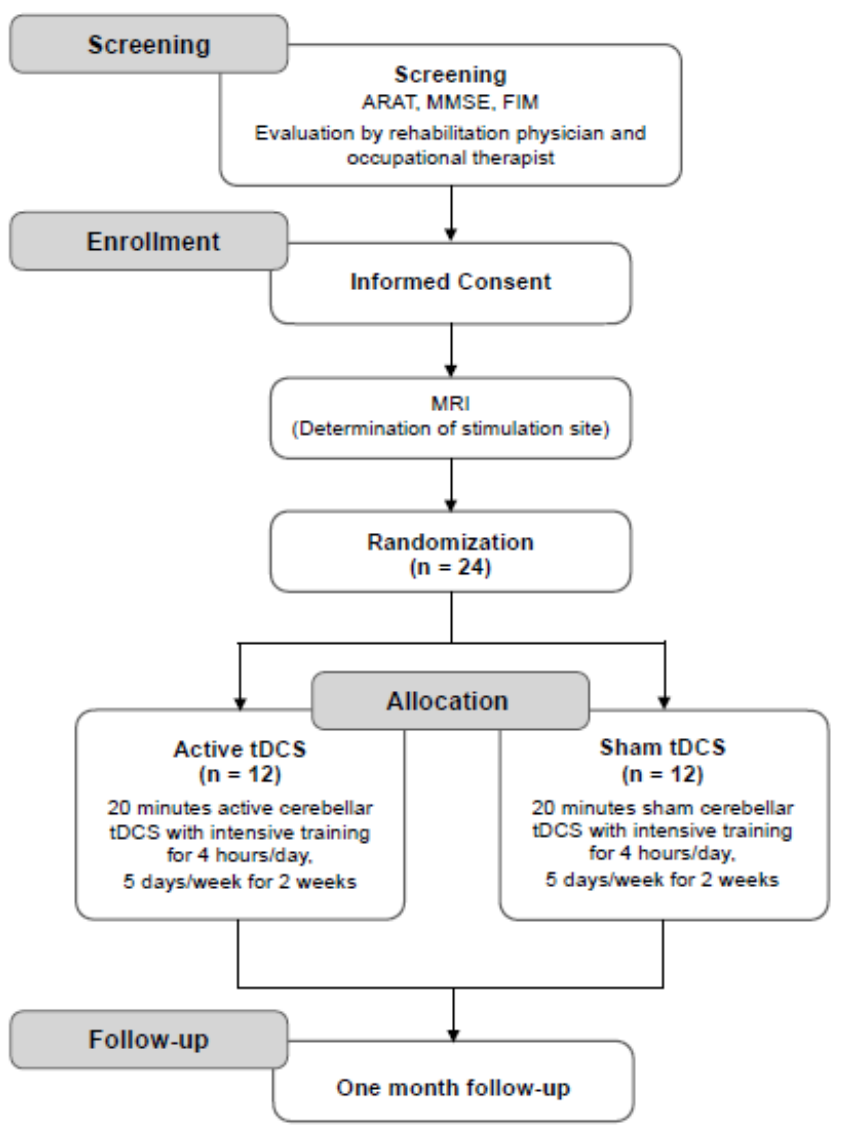

Figure 1

Study flowchart.

(A)

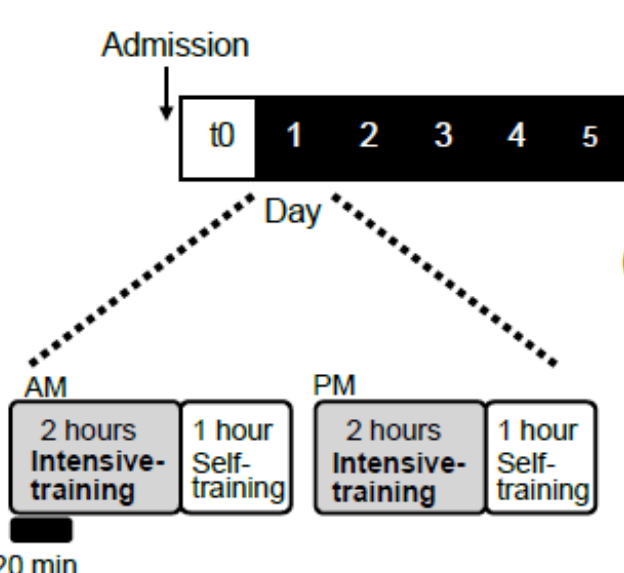

min

Active or sham-

tDCS

(C)

Active tDCS

Intensity

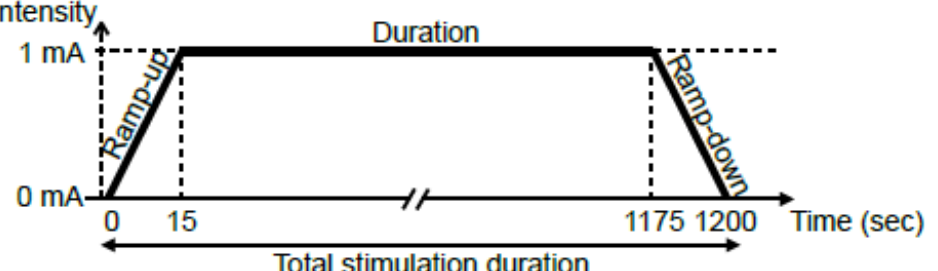

Sham tDCS

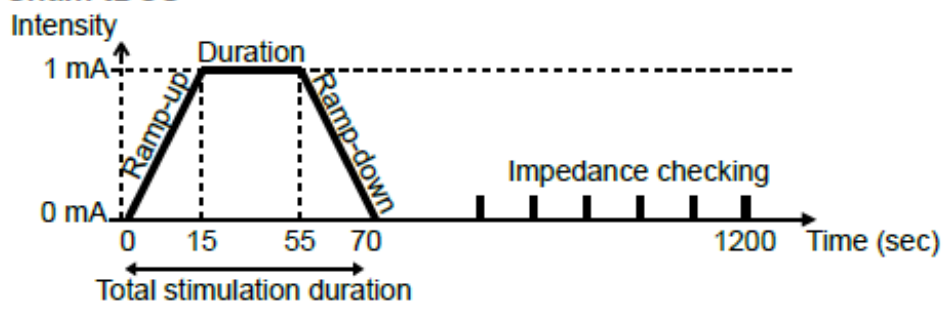

Figure 2 
Study protocol. (A) Schedule of the study. The intensive upper limb training and tDCS interventions will be repeated for 5 consecutive days per week for 2 weeks (i.e., 10 days of intervention). During the middle 2 days, the participants will engage only in the intensive training. Assessments will be conducted prior to the intervention ( $\mathrm{t} 0$, baseline), at the middle of the intervention ( $\mathrm{t} 1$, middle), immediately after the intervention (t2, post), and 1 month after the intervention ( $\mathrm{t} 3$, follow-up). (B) Training schedule per day. Participants will undergo intensive upper limb training for 4 hours per day ( 2 hours per half-day) and receive cerebellar tDCS (active or sham) for 20 min during the beginning of the training. (C) Time course of active (upper panel) and sham (bottom panel) tDCS conditions.

(A)

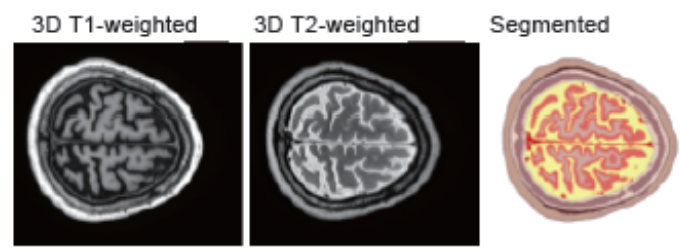

(B)
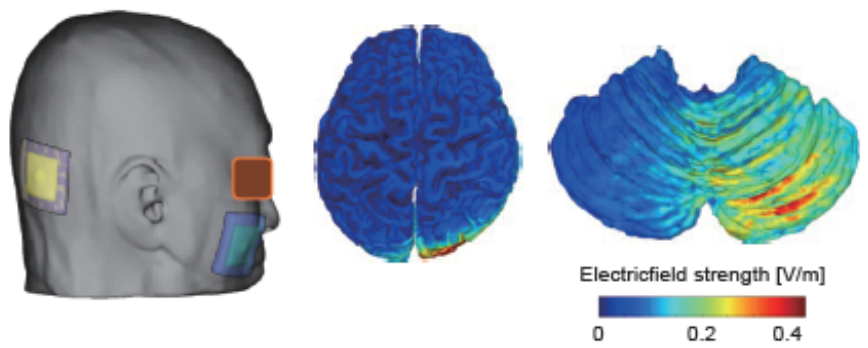

(C)
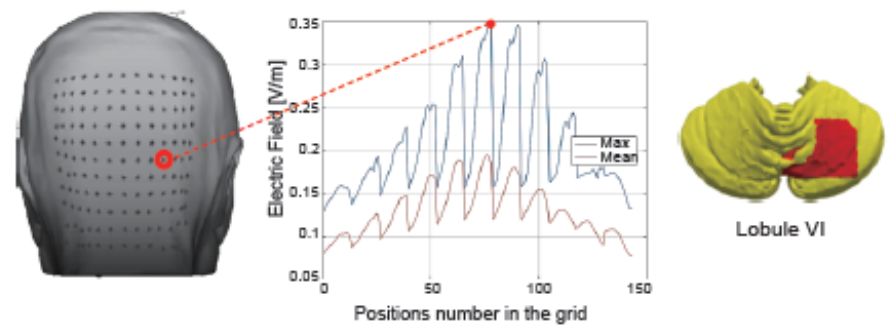

Figure 3

Computational method to determine the optimal individualized position of the tDCS montage. (A) Head model segmentation. Volume conductor of the head model of each patient will be constructed from the segmentation of MRI data. (B) Computational modeling of a representative example. The individualized electric field on the cerebellum using a bipolar montage (anode in yellow and cathode in blue) will be simulated. (C) Optimal montage position. The optimal location of the anode that maximizes the electric field strength (maximum and average) on the region of interest (lobule $\mathrm{VI}$ ) is selected from a grid of $13 \times 11$ positions. 


\begin{tabular}{|c|c|c|c|c|c|}
\hline & \multicolumn{5}{|c|}{ STUDY PERIOD } \\
\hline \multirow[b]{2}{*}{ TIMEPOINT } & \multirow{2}{*}{$\begin{array}{c}\text { Enrolment } \\
-t_{1}\end{array}$} & \multirow{2}{*}{$\frac{\text { Baseline }}{t_{0}}$} & \multicolumn{2}{|c|}{ Post-allocation } & \multirow{2}{*}{$\frac{\text { Follow-up }}{t_{3}}$} \\
\hline & & & $t_{1}$ & $t_{2}$ & \\
\hline \multicolumn{6}{|l|}{ ENROLMENT: } \\
\hline Eligibility screen & $x$ & & & & \\
\hline \multirow[t]{2}{*}{ Informed consent } & $x$ & & & & \\
\hline & & $x$ & & & \\
\hline \multicolumn{6}{|l|}{ INTERVENTIONS: } \\
\hline \multicolumn{6}{|l|}{$\begin{array}{r}\text { Active or sham } \\
\text { tDCS }\end{array}$} \\
\hline \multicolumn{6}{|l|}{$\begin{array}{r}\text { Intensive upper limb } \\
\text { training }\end{array}$} \\
\hline \multicolumn{6}{|l|}{ ASSESSMENTS: } \\
\hline $\begin{array}{c}\text { Demographic data } \\
\text { collection }\end{array}$ & $x$ & & & & \\
\hline MMSE & $x$ & & & & \\
\hline FIM & $x$ & & & & \\
\hline ARAT & $x$ & $x$ & $\mathrm{x}$ & $x$ & $x$ \\
\hline FMA & & $x$ & & $x$ & $x$ \\
\hline$M A L-14$ & & $x$ & & $\mathrm{x}$ & $x$ \\
\hline$C B I \cdot M E P$ & & $x$ & & $\mathrm{x}$ & $x$ \\
\hline MRI & & $x$ & & $\mathrm{x}$ & \\
\hline Safety & & & + & $\rightarrow$ & \\
\hline
\end{tabular}

Figure 4

SPIRIT figure. tDCS, transcranial direct current stimulation; MMSE, Mini-Mental State Examination; FIM, functional independence measure; ARAT, Action Research Arm Test; FMA, Fugl-Meyer assessment for the upper extremity; MAL-14, Motor Activity Log-14; CBI, cerebellar brain inhibition; MEP, motor-evoked potential; MRI, magnetic resonance imaging
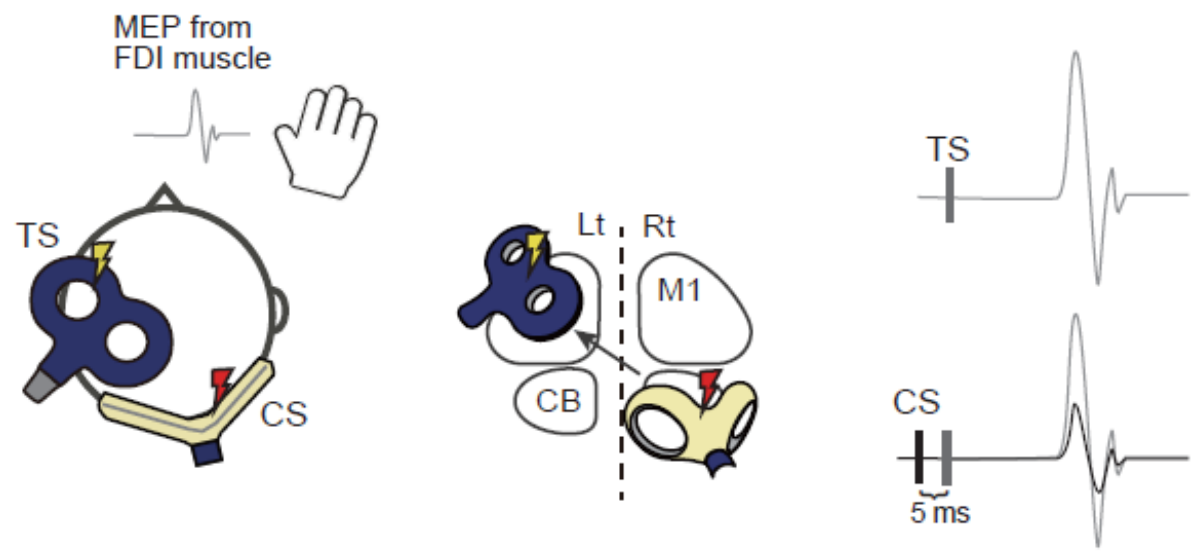

\section{Figure 5}

Position of the TMS coils for measuring CBI and MEP. A figure-of-eight coil will be placed over the ipsilesional side of M1 to deliver the test stimuli for eliciting MEP in the FDI muscle. A double-cone coil will be placed over the contralesional side of the cerebellum to deliver the CS. The CS will be delivered $5 \mathrm{~ms}$ prior to the TS. CBI will be calculated as the ratio between the peak-to-peak amplitude of the conditioned MEP (black line) and the unconditioned test MEP (grey line). MEP, motor-evoked potential; FDI, first dorsal 
interosseous; TS, test stimulation; $\mathrm{CS}$, conditioning stimulation; $\mathrm{M} 1$, primary motor cortex; $\mathrm{CBI}$, cerebellar brain inhibition; $\mathrm{CB}$, cerebellum 